\title{
Human trypsin inhibitor reduces the apoptosis of lipopolysaccharide-induced human kidney-2 cells by promoting mitochondrial fusion
}

\author{
NING LIU* , ZHIYI JIANG* , YONGJUN LIU, YAO NIE, JUAN CHEN, \\ BIN OUYANG, XIANGDONG GUAN and MINYING CHEN
}

Department of Surgical Intensive Care Unit of The First Affiliated Hospital of Sun Yat-sen University, Guangzhou, Guangdong 510080, P.R. China

Received July 3, 2016; Accepted May 2, 2017

DOI: $10.3892 / \mathrm{mmr} .2017 .6927$

\begin{abstract}
Imbalance in mitochondrial fusion/fission is one of the mechanisms leading to sepsis-induced mitochondrial dysfunction and cell apoptosis. The present study examined the effects of human trypsin inhibitor (UTI), a well-known antioxidant and anti-inflammatory substance, on mitochondrial dynamics and cell apoptosis in lipopolysaccharide (LPS)-induced human kidney-2 (HK-2) cells. The HK-2 cells were incubated for $24 \mathrm{~h}$ either with LPS $(800 \mathrm{ng} / \mathrm{ml})$ or LPS $(800 \mathrm{ng} / \mathrm{ml})$ mixed with UTI $(250 \mathrm{U} / \mathrm{ml})$. Cell viability was assessed using a3-(4,5-dimethyl-2-thiazolyl)-2, 5-diphenyl-2-H-tetrazolium bromide assay. Oxidative activities (estimated by maleic dialdehyde and superoxide dismutase), levels of inflammatory cytokines interleukin (IL)-6 and tumor necrosis factor (TNF)- $\alpha$, and levels of ATP were measured using an enzyme-linked immunosorbent assay. The expression
\end{abstract}

Correspondence to: Dr Minying Chen or Dr Xiangdong Guan, Department of Surgical Intensive Care Unit of The First Affiliated Hospital of Sun Yat-sen University, 58 Zhongshan Road, Guangzhou, Guangdong 510080, P.R. China

E-mail: chmy1969@hotmail.com

E-mail: carlg@163.net

${ }^{*}$ Contributed equally

Abbreviations: UTI, human trypsin inhibitor; LPS, lipopolysaccharide; AKI, acute kidney injury; HK-2, human kidney 2; TNF- $\alpha$, tumor necrosis factor- $\alpha$; IL-6, interleukin 6; MDA, maleic dialdehyde; SOD, superoxide dismutase; DAPK-2, death-associated protein kinase 2; Mfn1, mitofusin-1; Mfn2, mitofusin-2; MMP, mitochondrial membrane potential; Bcl-2, B-cell lymphoma-2; Bcl-xL, B-cell lymphoma-extra large; PARP, poly ADP-ribose polymerase; MTT, 3-(4, 5-dimethyl-2-thiazolyl)-2, 5-diphenyl-2-H-tetrazolium bromide; OD, optical density; ELISA, enzyme-linked immunosorbent assay; $\mathrm{NC}$, negative control

Key words: human trypsin inhibitor, apoptosis, mitochondrial dynamic, mitofusin-1, mitofusin-2, death-associated protein kinase 2 levels of the mitochondrial fission protein, death-associated protein kinase 2 (DAPK-2), mitofusin (Mfn)1 and Mfn2 mitochondrial fusion proteins, and apoptotic and anti-apoptotic biomarkers, including cytochrome $c$, caspase-3, caspase-9, B-cell lymphoma (Bcl)-2, Bcl-extra large and poly ADP-ribose polymerase (PARP), were assessed using western blot analyses. The changes in mitochondrial membrane potential were analyzed following JC-1 staining. Annexin V/propidium iodide assays were used to evaluate cell apoptosis. The results showed that the balance of mitochondrial dynamics was towards mitochondrial fusion in the UTI group, as a reduced expression of DAPK2, and increased expression levels of Mfn1 and Mfn2 were detected ( $\mathrm{P}<0.05$, vs. LPS group). In addition, adecline in the levels of the inflammatory cytokines, TNF- $\alpha$ and IL-6, and the oxidative activities, reflected by an increase in SOD and a decrease in MDA ( $\mathrm{P}<0.05$, vs. LPS group) were observed. Cell apoptosis was inhibited following co-treatment with UTI ( $<<0.05$, vs. LPS group). It was concluded that UTI may protect mitochondrial functions by promoting mitochondrial fusion and limiting mitochondrial fission, thus reducing the apoptosis of LPS-induced HK-2 cells.

\section{Introduction}

Acute kidney injury (AKI) is a common complication of sepsis, which increases mortality rates to as high as $70 \%$ (1). However, the pathophysiology of sepsis-induced AKI remains to be fully elucidated (2). Excessive fission and/or insufficient fusion of mitochondria, which contributes to the progression of sepsis, may be evoked by oxidative stress, and can lead to the loss of mitochondrial function and the apoptosis of tubular cells under stress $(3,4)$.

Human trypsin inhibitor (urinary trypsin inhibitor; UTI) is a Kunitz-type protease inhibitor, which inhibits the activity or release of lysosomal enzymes $(5,6)$. The effects of UTI include the protection of endothelial cells, suppression of excessive superoxide anion radicals, and reductions in systemic inflammation and oxidative stress (7). It has been reported that UTI can protect mitochondria from ischemia functionally and morphologically, thus benefiting renal function $(8,9)$. However, the mechanism remains to be elucidated. 
In the present study, it was hypothesized that UTI may have beneficial effects on regulating mitochondrial dynamics. To confirm this hypothesis, the levels of the mitochondrial fission protein, death-associated protein kinase 2 (DAPK-2), and two types of mitochondrial fusion proteins, mitofusin-1 (Mfn1) and mitofusin-2 (Mfn2), were examined in lipopolysaccharide (LPS)-induced human kidney-2 (HK-2) cells incubated with or without UTI. The oxidative activities of inflammatory cytokines, indicated by maleic dialdehyde (MDA) and superoxide dismutase (SOD), and cell apoptosis were also measured.

\section{Materials and methods}

Drugs, reagents and kits. LPS from Escherichia coli 055:B5 and 3-(4,5-dimethyl-2-thiazolyl)-2,5-diphenyl-2-H-tetrazolium bromide (MTT) were obtained from Sigma-Aldrich; Merck Millipore (Darmstadt, Germany). UTI was purchased from Techpool Bio-pharma Co., Ltd. (Guangzhou, China). MitoProbe J-aggregate (JC-1; 5,5',6,6'-tetrachloro-1,1',3,3'tetraethyl-benzimidazolyl-carbocyanineiodide), propidium iodide (PI), the Annexin V-FITC Apoptosis Detection kit, SOD assay kit (cat. no. KGT00150-1) and MDA assay kit (cat. no. KGT004) were obtained from Nanjing KeyGen Biotech Co., Ltd. (Nanjing, China). The ATPlite 1 step Luminescence ATP Detection Assay system was obtained from PerkinElmer, Inc. (Waltham, MA, USA). The following primary antibodies were used: Caspase-3 (cat. no. SC-7272; 1:1,000; Santa Cruz Biotechnology, Inc., Dallas, TX, USA), caspase-9 (cat. no. 9502S; 1:1,000; CST Biological Reagents Company Ltd., Shanghai, China), PARP (cat. no. ab96476; 1:1,000; Abcam, Cambridge, UK), B-cell lymphoma (Bcl)-2 (cat. no. 2870S; 1:1,000; CST Biological Reagents Company Ltd.), Bcl-extra large (Bcl-xL; cat. no. 2764; 1:1,000; CST Biological Reagents Company Ltd.), $\beta$-actin (cat. no. AP0060; 1:3,000; Bioworld Technology, Inc., St Louis Park, MN, USA), DAPK-2 (cat. no. 3432-1; 1:1,000; Epitomics; Abcam), Mfn1 (cat. no. 5870-1; 1:1,000; Epitomics; Abcam) and Mfn2 (cat. no. 3272-1; 1:1,000; Epitomics; Abcam). Secondary antibody (horseradish peroxidase-labeled goat anti-mouse immunoglobulin G) was obtained from Abgent Biotech Co., Ltd. (cat. no. LP1002a; 1:10,000; Suzhou, China).

Cell culture. The HK-2 cells, an immortalized proximal tubular epithelial cell line from the normal adult human kidney (10), were obtained from American Type Culture Collection (Manassas, VA, USA). The HK-2 cells were cultured in Dulbecco's modified Eagle's medium F-12 (Hyclone; GE Healthcare Life Sciences, Logan, UT, USA; cat. no. SH30023.01) supplemented with $10 \%$ fetal bovine serum (cat. no. 10099-141; Gibco; Thermo Fisher Scientific, Inc., Waltham, MA, USA) in an atmosphere of $5 \% \mathrm{CO}_{2}$ at $37^{\circ} \mathrm{C}$.

LPS administration. This procedure was performed to determine the appropriate concentration of LPS to use in the experiments. The cell culture medium was replaced with complete medium supplemented with $0.5 \%$ fetal bovine serum and various concentrations of LPS $(0,100,200,400,800,1,600$ and 3,200 $\mathrm{ng} / \mathrm{ml})$. Each sample was incubated for different durations $(3,6,12$ and $24 \mathrm{~h})$ at a density of 2,500 cells/well at $37^{\circ} \mathrm{C}$, which was performed three times.
Cell viability assay. The cell viability was assessed using a commercial MTT-based assay. This assay detects viable cells based on the production of the purple compound, formazan, in viable cells. Following incubation with LPS or LPS+UTI, $10 \mu \mathrm{l}$ of MTT $(5 \mathrm{mg} / \mathrm{ml})$ was added to each well, and the plates were incubated at $37^{\circ} \mathrm{C}$ for $4 \mathrm{~h}$. The content of the wells was eluted and the precipitate was dissolved with $150 \mu \mathrm{l}$ of MTT solubilization reagent (dimethyl sulfoxide). The optical density value (OD value) was read at a wavelength of $490 \mathrm{~nm}$ using the Model 680 Microplate Reader (Bio-Rad Laboratories, Inc., Hercules, CA, USA). Cell viability was determined as the ratio of surviving cells in each group divided by the number in the control ethanol-treated group. The LPS concentration shown to reduce cell viability the most, but affect the surrounding environment of the cells the least, was selected.

UTI administration. This procedure was performed to determine the appropriate concentrations of UTI to use in the experiments. The cell culture media were replaced with complete media supplemented with $10 \%$ fetal bovine serum containing LPS at a final concentration of $800 \mathrm{ng} / \mathrm{ml}$ (based on the results of the LPS intervention experiment) and various concentrations of UTI $(100,150,200,250,300,350$ and $400 \mathrm{U} / \mathrm{ml})$. In the control group, neither LPS nor UTI was administered. The cell viability assay was then repeated for selection of the UTI concentrations causing a significant increase in cell viability.

Groups. Based on the results of the UTI intervention experiment, the HK-2 cells were randomly divided into a control group, LPS group and UTI group. The cells were treated with LPS at a final concentration of $800 \mathrm{ng} / \mathrm{ml}$ in the LPS group, and $800 \mathrm{ng} / \mathrm{ml}$ LPS together with $250 \mathrm{U} / \mathrm{ml}$ UTI in the UTI group. All cells were cultured at a density of 2,500 cells/well and maintained at $37^{\circ} \mathrm{C}$ for $24 \mathrm{~h}$.

Measurement of inflammatory response and oxidative activity. Enzyme-linked immunosorbent assay (ELISA) kits were used in this procedure. Following treatment of cells with LPS and UTI, as described above, the cell supernatants were collected (centrifuged at $12,000 \mathrm{x} \mathrm{g}$ for $5 \mathrm{~min}$ at $37^{\circ} \mathrm{C}$ ) and stored at $-80^{\circ} \mathrm{C}$. The levels of inflammatory cytokines interleukin (IL)-6 and tumor necrosis factor- $\alpha(\mathrm{TNF}-\alpha)$ were measured to evaluate the inflammatory response. SOD and MDA were measured to estimate the oxidative activities. In all cases, a standard curve was constructed from standards provided by the manufacturer.

Analysis of mitochondrial fission/fusion and apoptotic proteins. Western blot analysis was performed to detect the levels of total proteins. Following treatment with LPS and UTI, as described above, the cells were lysed using radioimmunoprecipitation assay buffer $[0.6 \%$ SDS, $4 \%$ glycerine, $12.5 \%$ Tris- $\mathrm{Hcl}(1 \mathrm{M} ; \mathrm{pH} 6.8), \mathrm{H}_{2} \mathrm{O}$ ], and the concentrations of total proteins in each sample were measured using a BCA protein assay kit. The proteins (50 $\mu \mathrm{g} /$ lane) were separated using SDS-PAGE on a $10 \%$ gel and transferred onto PVDF membranes; the membranes were then blocked in 5\% fat-free milk at room temperature for $2 \mathrm{~h}$. Following incubation with primary antibodies against caspase-3, caspase-9, PARP, Bcl-2, 
Bcl-xL and $\beta$-actin, and primary antibodies against DAPK-2, Mfn 1 and Mfn 2 at a dilution of 1:1,000 at $4^{\circ} \mathrm{C}$ overnight, the membranes were probed with goat-anti-mouse secondary antibodies at a dilution of $1: 10,000$ at $37^{\circ} \mathrm{C}$ for $1 \mathrm{~h}$. The signals were detected and then analyzed using SensiAnsys software (version JS-680D; Shanghai Peiqing Science \& Technology Co., Ltd., Shanghai, China).

Mitochondrial membrane potential (MMP) analysis. The fluorescent probe JC-1 was used to detect the MMP. Following treatment of the cells with LPS and UTI, as described above, the culture medium was removed and $1-2 \times 10^{6}$ cells were harvested by trypsinization with $0.25 \%$ Trypsin-EDTA. The cells were washed twice with PBS and centrifuged at $170 \mathrm{x} g$ for $5 \mathrm{~min}$ at room temperature. For each sample, the cells were suspended in $500 \mu 1$ of JC-1 stock solution (final concentration, $2 \mathrm{mg} / \mathrm{ml}$; diluted by $1 \mathrm{X}$ incubation buffer) and then incubated for $15-20 \mathrm{~min}$ at $37^{\circ} \mathrm{C}$. The staining procedure was performed according to the manufacture's protocol. The stained cells were then centrifuged at $700 \mathrm{x}$ g for $5 \mathrm{~min}$ at room temperature, washed twice in $1 \mathrm{X}$ incubation buffer and re-suspended in $500 \mu 1 \mathrm{lX}$ incubation buffer. JC-1 aggregates (red fluorescence) favor high MMP intact cells and, in response to the loss of MMP, JC-1 monomers are formed showing green fluorescence. The percentage of cells showing a decrease in red fluorescence was evaluated to determine the extent of depolarization. The fluorescence intensity of the $\mathrm{red} /$ green ratio was determined semi-quantitatively using flow cytometry (Beckman Coulter, Inc., Brea, CA, USA). Cells with collapsed MMPs exhibited a decrease in the red/green fluorescence intensity ratio.

Measurement of intracellular ATP. Intracellular levels of ATP were measured using a luminescence ATP detection assay according to the manufacturer's protocol. Following treatment of the cells with LPS and UTI, as described above, and equilibrating the microplate at room temperature for $30 \mathrm{~min}, 100 \mu \mathrm{l}$ of the reconstituted lyophilized substrate solution was added to each well. Each lyophilized substrate solution vial was reconstituted with $10 \mathrm{ml}$ substrate buffer and agitated gently until homogenous. The 96-well microplate was then shaken for $5 \mathrm{~min}$, and the relative light unit (RLU) was measured using the JANUS ${ }^{\circledR}$ Automated Workstation (PerkinElmer, Inc.) within $10 \mathrm{~min}$ in the dark.

Cell apoptosis assay. The Annexin V-FITC kit was used to detect the externalization of phosphatidylserine of the cell membrane, one of the typical markers of early apoptosis. Following treatment of cells with LPS and UTI as described above, the culture medium was removed and $1-5 \times 10^{5}$ cells were harvested by trypsinization without Trypsin-EDTA, washed twice with PBS and centrifuged at $700 \mathrm{x}$ g for $10 \mathrm{~min}$ at room temperature. Then the cells were re-suspended in $500 \mu \mathrm{l}$ of binding buffer. Subsequently, $5 \mu \mathrm{l}$ Annexin V-FITC and $5 \mu \mathrm{l}$ of propidium iodide (PI) were added into each $500 \mu \mathrm{l}$ of solution according to the manufacturer's protocol. The cells were then gently vortexed and incubated for 5-15 min at room temperature in the dark. Flow cytometry (Beckman Coulter, Inc.) was used to analyze the samples within $1 \mathrm{~h}$. The excitation wavelength was $488 \mathrm{~nm}$ and the emission wavelength was $530 \mathrm{~nm}$. The percentage of Annexin V(+)/PI(-) and Annexin V(+)/PI(+) cells represent the ratios of early and late stage of apoptosis respectively.

Statistical analysis. All experiments were performed three times. All numerical data are expressed as the mean \pm standard deviation. Multiple comparison between the groups was performed using one-way analysis of variance and the least significant difference method. SPSS 22.0 software (IBM SPSS, Armonk, NY, USA) was used. $\mathrm{P}<0.05$ was considered to indicate a statistically significant difference. All statistical graphs were produced using OriginPro 9.0 (OriginLab Corporation, Northampton, MA, USA).

\section{Results}

Cell viability. As shown in Fig. 1A, cell viability was reduced as LPS concentration increased. LPS at concentrations of 1,600 and 3,200 $\mathrm{ng} / \mathrm{ml}$ caused a rapid reduction in cell viability in all groups, which may have resulted from the high osmotic pressure induced by the high drug concentration on the surrounding environment of the cells. In the 3 and $6 \mathrm{~h}$ groups, no significant changes in cell viability were observed with LPS concentrations of $50-800 \mathrm{ng} / \mathrm{ml}$. In the 12 and $24 \mathrm{~h}$ groups, cell viability was significantly decreased with LPS concentrations of $50-800 \mathrm{ng} / \mathrm{ml}(\mathrm{P}<0.05$, vs. control) the lowest level was observed at $800 \mathrm{ng} / \mathrm{ml}$ in the $24 \mathrm{~h}$ group. Based on these results, the HK-2 cells were treated with LPS at $800 \mathrm{ng} / \mathrm{ml}$ for $24 \mathrm{~h}$ in the subsequent experiments.

As shown in Fig. 1B, following co-treatment with different concentrations of UTI for $24 \mathrm{~h}$, cell viability was positively correlated with drug concentrations at 100-200 U/ml, but decreased rapidly at concentrations $>250 \mathrm{U} / \mathrm{ml}$, which suggested that concentrations of UTI $>250 \mathrm{U} / \mathrm{ml}$ led to an excessive increase in osmotic pressure. No significant differences in cell viability were observed between concentrations of UTI at 250 and $200 \mathrm{U} / \mathrm{ml}$. Therefore, $250 \mathrm{U} / \mathrm{ml}$ of UTI was used to evaluate its protective function in subsequent experiments.

Release of inflammatory cytokines and oxidative factors induced by LPS is reduced by UTI. As shown in Fig. 2A and B, following treatment with LPS for $24 \mathrm{~h}$, significant increases in the release of IL- 6 and TNF- $\alpha$ were detected in the HK-2 cells $(\mathrm{P}<0.05$, vs. control). In the UTI group, the increase in these two inflammatory cytokines were significantly lower $(\mathrm{P}<0.05$ vs. LPS group). The oxidation product, MDA, was significantly increased, whereas the antioxidant enzyme, SOD, was decreased following treatment with LPS ( $\mathrm{P}<0.05$ vs. control). These effects were less marked in the UTI group $(\mathrm{P}<0.05$ vs. LPS group; Fig. 2C and D).

LPS-induced increase in mitochondrial fission and reduction in mitochondrial fusion are reversed by UTI. As shown in Fig. 3, the expression of DAPK2 was increased, and the levels of Mfn1 and Mfn2 were decreased in the LPS group $(\mathrm{P}<0.05$ vs. control). These changes suggested that LPS induced increased fission and weakened fusion of mitochondria. In the cells co-treated with UTI, decreased expression of DAPK2, and enhanced expression of Mfn1 and Mfn2 were 

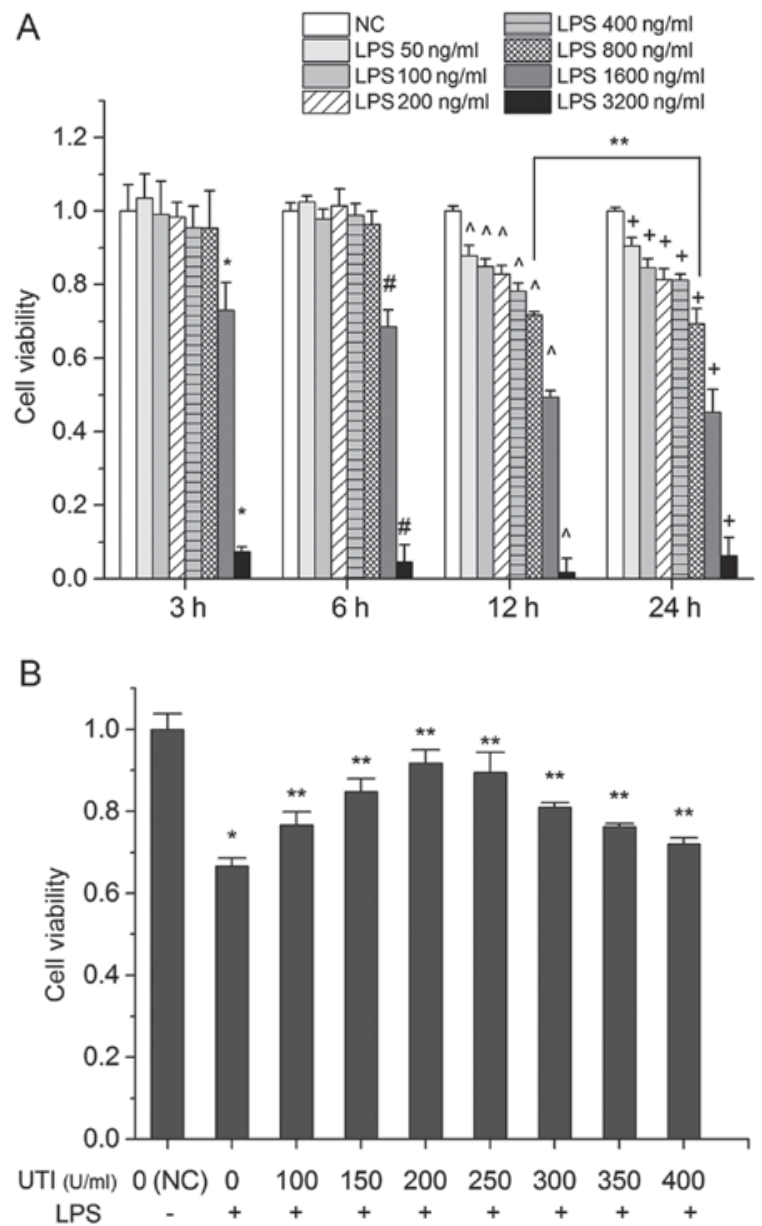

Figure 1. (A) Effects of LPS on HK-2 cells. Cell viability is presented as relative MTT activity and determined as $1-\mathrm{OD}_{\mathrm{LPS}} / \mathrm{OD}_{\mathrm{NC}}$. Cell viability decreased as LPS concentration increased. When treated with LPS for 3 and $6 \mathrm{~h}$, no significant change in cell viability was observed. When treated with LPS at 1,600 and $3,200 \mathrm{mmol} / 1$, cell viability was markedly reduced. Values are expressed as the mean \pm standard deviation of triplicate experiments. ${ }^{*} \mathrm{P}<0.05$, vs. NC (3 h), ${ }^{\#} \mathrm{P}<0.05$, vs. NC (6 h), ${ }^{\wedge} \mathrm{P}<0.05$, vs. NC (12 h), ${ }^{+} \mathrm{P}<0.05$, vs. NC $(24 \mathrm{~h}),{ }^{* *} \mathrm{P}<0.05$ between groups. (B) Effects of UTI co-treated with LPS $(800 \mathrm{ng} / \mathrm{ml})$ in HK-2 cells. Cell viability was presented as relative MTT activity and determined as $1-\mathrm{OD}_{\mathrm{UTI}} / \mathrm{OD}_{\mathrm{NC}}$. Cell viability was positively correlated with UTI concentrations of 100-200 U/ml, whereas a rapid decrease was observed at concentrations $>250 \mathrm{U} / \mathrm{ml}$. The data are presented as the mean \pm standard deviation of triplicate experiments. ${ }^{*} \mathrm{P}<0.05$, vs. $\mathrm{NC}$; ${ }^{* *} \mathrm{P}<0.05$, vs. LPS group. LPS, lipopolysaccharide; UTI, human trypsin inhibitor; NC, negative control; OD, optical density; MTT, 3-(4, 5-dimethyl-2-thiazolyl)-2, 5-diphenyl-2-H-tetrazolium bromide.

found, compared with the levels in the UTI group $(\mathrm{P}<0.05$, vs. LPS group). These changes indicated that UTI increased mitochondrial fusion and limited mitochondrial fission.

LPS-induced decreases in MMP and intracellular ATP are reversed by UTI. As shown in Fig. 4A and B, LPS stimulation led to fewer high red JC-1-positive cells $(69.4 \pm 0.75 \%)$ compared with the control $(98.1 \pm 0.3 ; \mathrm{P}<0.05)$, which indicated a decrease in MMP. The decrease in MMP was less marked in the UTI group, compared with that in the LPS group ( $97.23 \pm 0.25$ vs. $69.4 \pm 0.75 \%$; $\mathrm{P}<0.05)$, which indicated amore stabilized mitochondrial membrane. As presented in Fig. 4C, RLU was significantly decreased following treatment with LPS for $24 \mathrm{~h}(0.12 \pm 0.05$ vs. $0.33 \pm 0.03$; $\mathrm{P}<0.05)$, compared with that in the negative control, and this reduction was
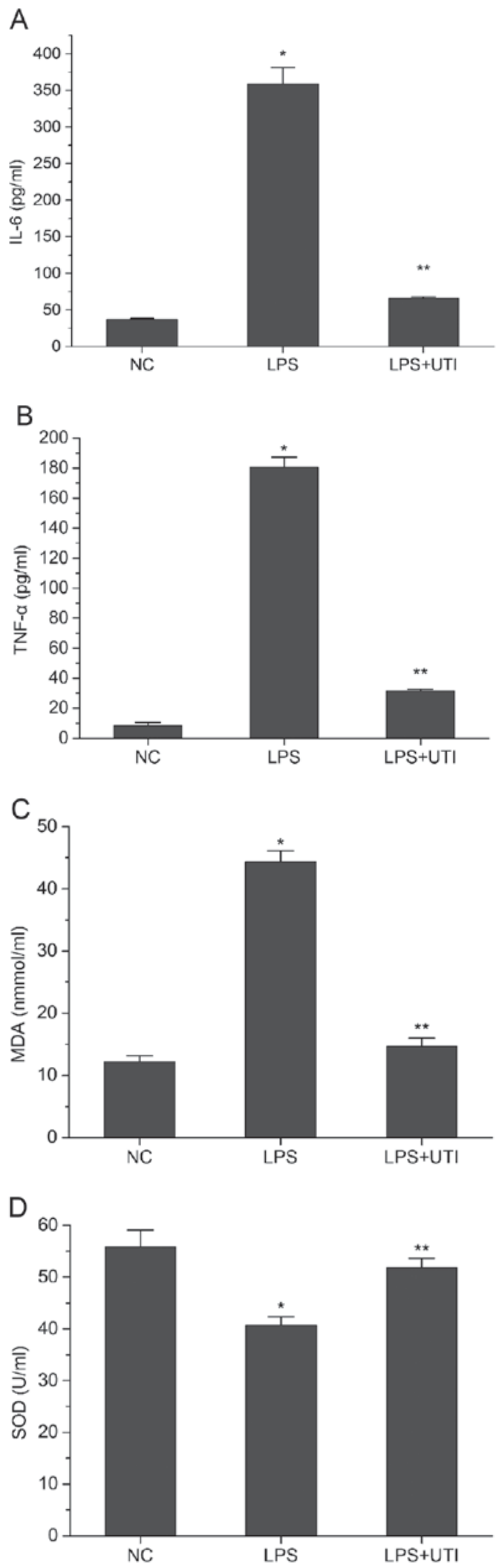

Figure 2. Release of inflammatory cytokines and oxidative factors were reduced by UTI. Expression of inflammatory cytokines (A) IL-6, (B) TNF- $\alpha$, the (C) oxidation product MDA and (D) antioxidant SOD were detected using enzyme-linked immunosorbent assay kits. Expression levels of IL-6 and TNF- $\alpha$ were lowerin the UTI group, compared with those in the LPS group. Expression of MDA was higherand that of SOD was lowerin the UTI group. Data are presented as the mean \pm standard deviation of triplicate experiments. ${ }^{*} \mathrm{P}<0.05$, vs. NC; ${ }^{* *} \mathrm{P}<0.05$, vs. LPS group. LPS, lipopolysaccharide; UTI, human trypsin inhibitor; IL-6, interleukin-6; TNF- $\alpha$, tumor necrosis factor- $\alpha$; NC, negative control; MDA, maleic dialdehyde; SOD, superoxide dismutase. 
A

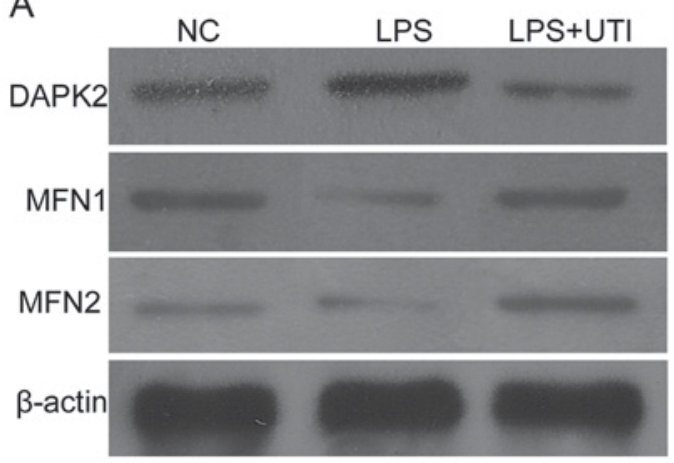

B

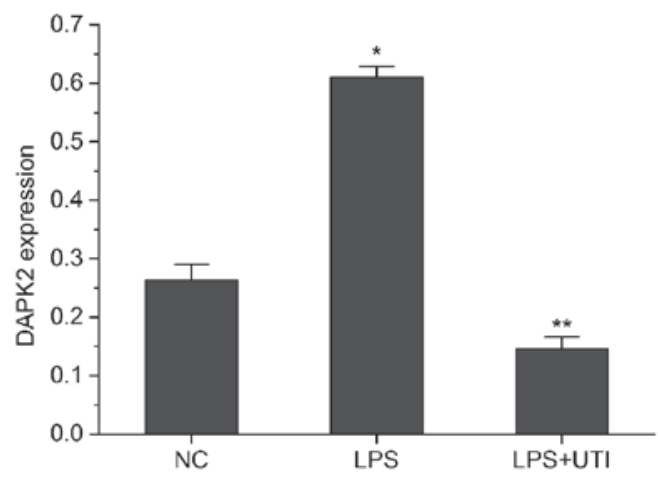

C

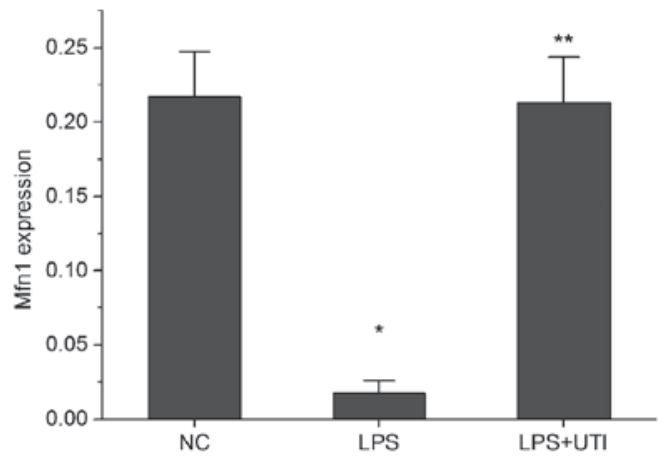

D

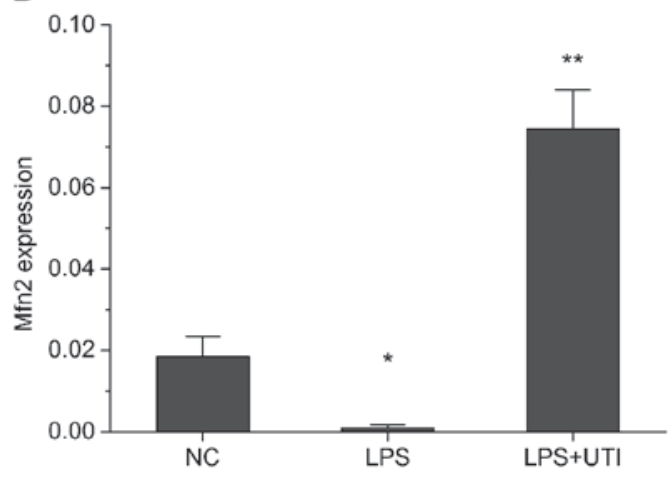

Figure 3. Co-treatment with UTI increases mitochondrial fusion and limits mitochondria fission in HK-2 cells. (A) Expression levels of DAPK2, Mfn1 and Mfn2 were analyzed using immunoblotting. Expression levels of (B) DAPK2, (C) Mfn1 and (D) Mfn2 were quantitatively determined using densitometry. The expression of DAPK2 increased in the LPS group and decreased in the UTI group. (C) Expression of Mfn1 decreased in the LPS group and increased in the UTI group. (D) Expression of Mfn2 decreased in the LPS group and increased in the UTI group. Data are presented as the mean \pm standard deviation of triplicate experiments. ${ }^{*} \mathrm{P}<0.05$, vs. NC; ${ }^{* *} \mathrm{P}<0.05$, vs. LPS group. LPS, lipopolysaccharide; UTI, human trypsin inhibitor; DAPK, death-associated protein kinase 2; Mfn, mitofusin; NC, negative control.
A
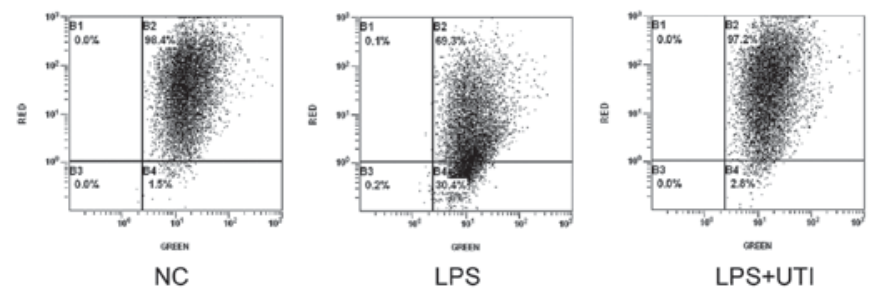

LPS+UTI

B

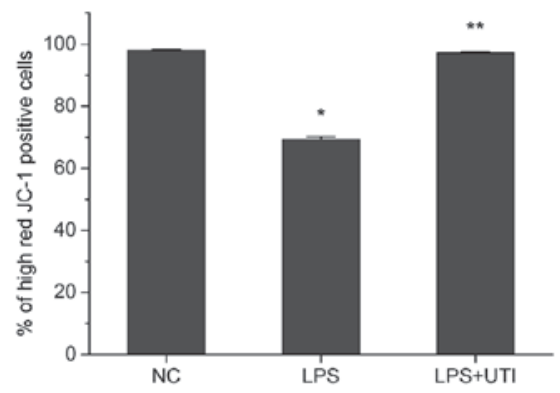

C

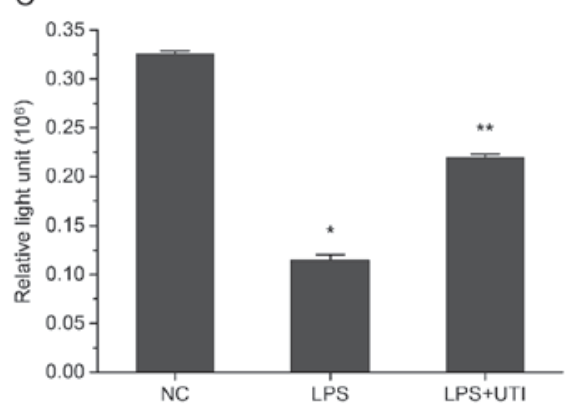

Figure 4. Decreases in MMP and intracellular levels of ATP caused by LPS are reversed by UTI. (A) Results of fluorescent probe JC-1 assay. (B) MMP was determined as the percentage of cells with high red fluorescence intensity. MMP in the UTI group was decreased, compared with that in the LPS group. Data are presented as the mean \pm standard deviation of triplicate experiments. ${ }^{*} \mathrm{P}<0.05$, vs. $\mathrm{NC} ;{ }^{* *} \mathrm{P}<0.05$, vs. LPS group. (C) Intracellular levels of ATP are presented as relative light units. The decrease of intracellular ATP was reduced by UTI. ATP levels were reduced following LPS treatment and increased in the UTI groups. Data are presented as the mean \pm standard deviation of triplicate experiments. ${ }^{*} \mathrm{P}<0.05$, vs. NC; ${ }^{* *} \mathrm{P}<0.05$, vs. LPS group. LPS, lipopolysaccharide; UTI, human trypsin inhibitor; MMP, mitochondrial membrane potential.

reversed in the UTI group, compared with that in the LPS group $(0.22 \pm 0.04$ vs. $0.12 \pm 0.05 ; \mathrm{P}<0.05)$. These results indicated that co-treatment with UTI prevented the mitochondrial dysfunction caused by LPS.

LPS-induced cell apoptosis is inhibited by UTI. As shown in Fig. 1B, cell viability was preserved in the UTI groups, compared with that in the LPS group $(\mathrm{P}<0.05)$, although UTI concentrations $>250 \mathrm{U} / \mathrm{ml}$ led to a descending trend of cell viability. This suggested that co-treatment with UTI prevented the cell apoptosis induced by LPS.

The hypothesis of the present study was confirmed using Annexin V and PI staining. As shown in Fig. 5, the increase in the percentage of Annexin $\mathrm{V}$-positive cells was marked in the LPS group (34.42 $\pm 0.64 \%)$, compared with that in the negative control $(6.47 \pm 0.17 ; \mathrm{P}<0.05)$, whereas cell apoptosis was inhibited following co-treatment with UTI (10.52 \pm 0.24$)$, compared with the LPS group $(34.42 \pm 0.64 \%$; $\mathrm{P}<0.05)$. 


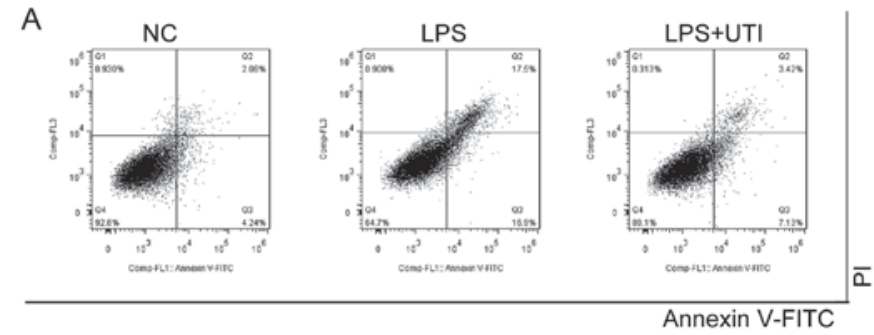

B

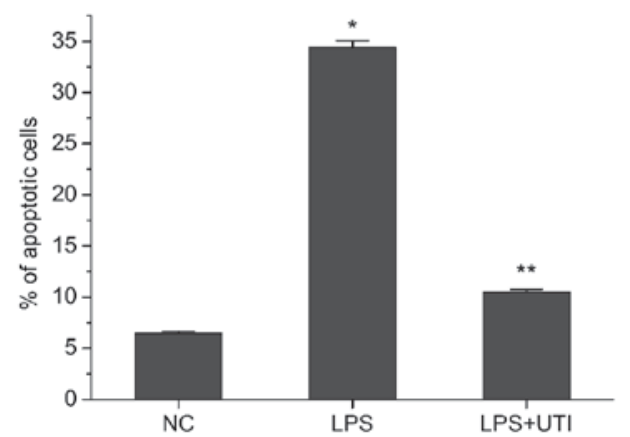

Figure 5. Apoptosis of LPS-induced HK-2 cells is inhibited by co-treatment with UTI. (A) Results of Annexin V-FITC assay. Percentages of Annexin $\mathrm{V}(+) / \mathrm{PI}(-)$ and Annexin $\mathrm{V}(+) / \mathrm{PI}(+)$ cells represent the ratios of early and late stage of apoptosis. (B) Cell apoptosis increased significantly in the LPS group and decreased in the UTI group. Data are presented as the mean \pm standard deviation of triplicate experiments. "P<0.05, vs. NC; ${ }^{* *} \mathrm{P}<0.05$, vs. LPS group. LPS, lipopolysaccharide; UTI, human trypsin inhibitor; NC, negative control; PI, propidium iodide.

As shown in Fig. 6, significantly higher expression levels of cytochrome $c$, caspase- 3 and caspase- 9 were observed in the HK-2 cells following incubation with LPS for $24 \mathrm{~h}(\mathrm{P}<0.05$, vs. control), whereas lower expression levels of cytochrome $c$, caspase- 3 and caspase- 9 were observed in the LPS+UTI group. In addition, as shown in Fig. 7A-C, the protein levels of Bcl-2 and $\mathrm{Bcl}-\mathrm{xL}$ were lower in HK-2 cells treated with LPS $(\mathrm{P}<0.05$ vs. control), where as their expression levels were increased in the LPS+UTI group $(\mathrm{P}<0.05)$. As shown in Fig. 7D, cleavage of PARP was reduced in the LPS group (Fig. 7D; P<0.05, vs. control), but levels of PARP were increased in the UTI group $(\mathrm{P}<0.05$, vs. LPS group).

\section{Discussion}

In previous studies, it was found that neither hemodynamic failure nor ischemia were the primary pathogenic factors in septic renal damage. Lerolle et al analyzed postmortem histopathological findings of 19 patients with septic AKI and reported that tubular cell apoptosis was the major histological abnormality. Even in patients with severe septic shock, tubular necrosis was minimal (11). It was reported that inflammation and renal cellular apoptosis were the essential factors $(12,13)$.

Mitochondria are dynamic organelles, which constantly undergo fission and fusion, during which they may exhibit a tubular or fragmented morphotype, or they may be assembled into networks in response to cellular energy demands and environmental challenges $(14,15)$. In mammalian cells, DAPK2 (also known as Drp1) is one of the primary mitochondrial fission proteins, and the large mitochondrial GTPases, Mfn1 and Mfn2 are important in mitochondrial fusion (16).
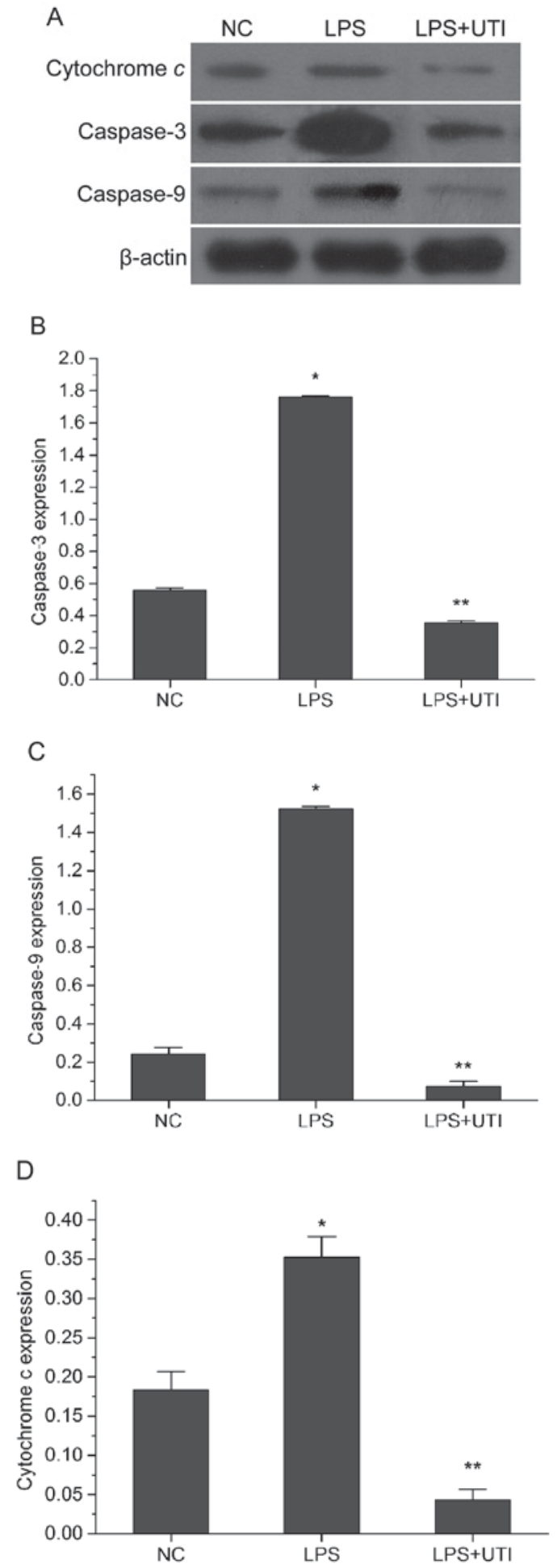

Figure 6. Expression levels of caspase-3, caspase-9 and cytochrome $c$ in LPS-induced HK-2 cells are downregulated by co-treatment with UTI. (A) Expression levels of caspase-3, caspase-9 and cytochrome $c$ were analyzed using immunoblotting. The expression ratios of (B) caspase-3, (C) caspase-9 and (D) cytochrome $c$ were quantitatively determined using densitometry. The expression of (B) caspase-3, (C) caspase-9 and (D) cytochrome $c$ increased following treatment with LPS. In the UTI group, expression levels were decreased. Data are presented as the mean \pm standard deviation of triplicate experiments. ${ }^{*} \mathrm{P}<0.05$, vs. $\mathrm{NC}$; ${ }^{* *} \mathrm{P}<0.05$, vs. LPS group, LPS, lipopolysaccharide; UTI, human trypsin inhibitor; NC, negative control.

The suppression of Drp-1 has been reported to maintain mitochondrial function and reduce the apoptosis of tubular 
A

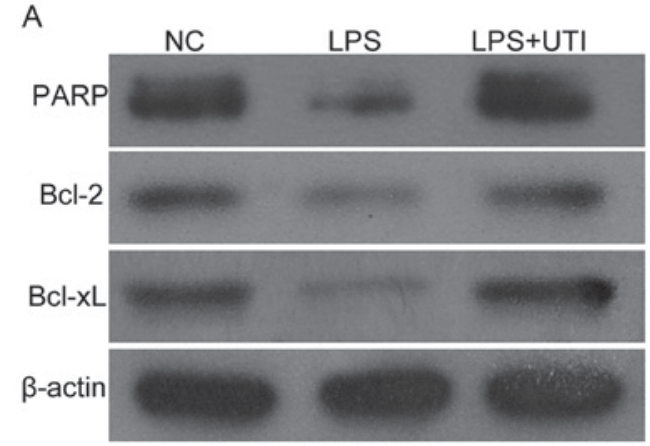

B

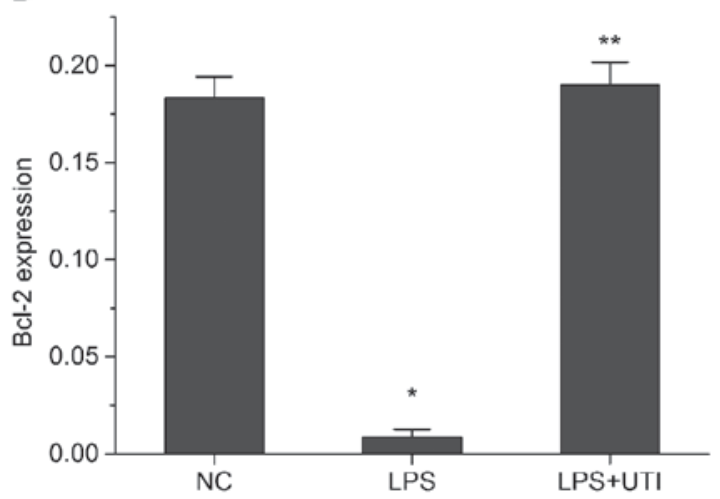

C
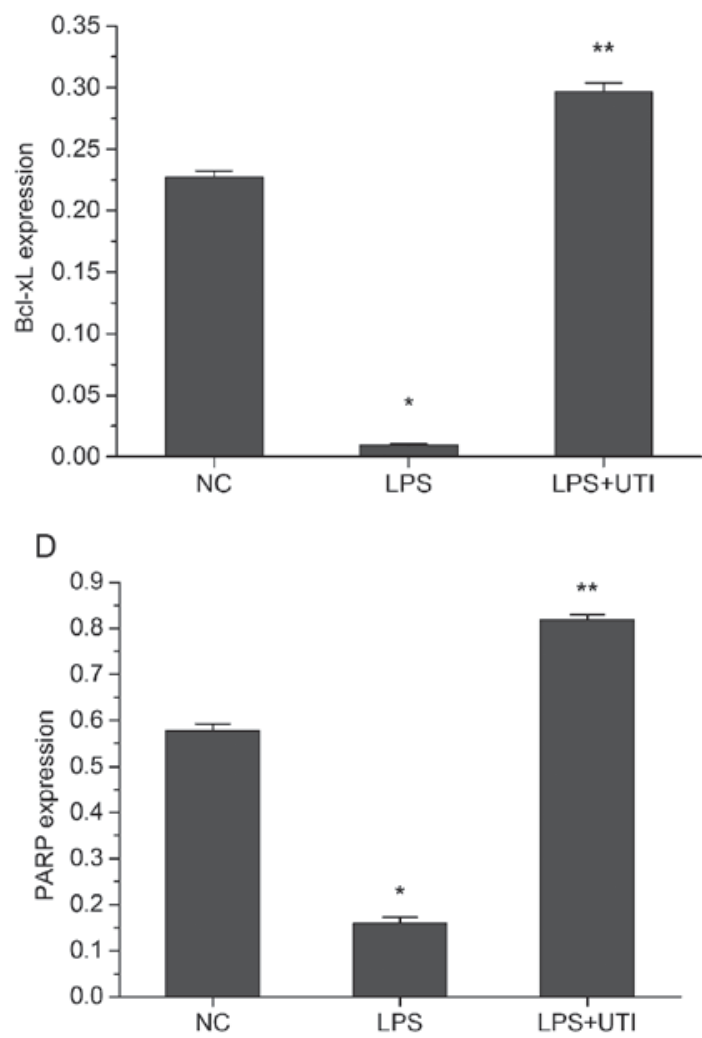

Figure 7. Expression levels of Bcl-2, Bcl-xL and PARP in LPS-induced HK-2 cells are upregulated by co-treatment with UTI. (A) Expression levels of $\mathrm{Bcl}-2, \mathrm{Bcl}-\mathrm{xL}$ and PARP were analyzed using immunoblotting. The ratios of (B) Bcl-2, (C) Bcl-xL and (D) PARP were quantitatively determined using densitometry. The expression levels of (B) Bcl-2, (C) Bcl-xL and (D) PARP were decreased following treatment with LPS. In the UTI group, expression levels were increased. Data are presented as the mean \pm standard deviation of triplicate experiments. ${ }^{*} \mathrm{P}<0.05$, vs. $\mathrm{NC}$; ${ }^{* *} \mathrm{P}<0.05$, vs. LPS group. LPS, lipopolysaccharide; UTI, human trypsin inhibitor; NC, negative control Bcl-2, B-cell lymphoma-2; Bcl-xL, Bcl-extra large; PARP, poly ADP-ribose polymerase. cells induced by ischemic injury, rhabdomyolysis and cisplatin nephrotoxin $(17,18)$. MFN2 deficiency has been shown to promote Bcl-2-associated $\mathrm{X}$ protein-mediated mitochondrial outer membrane injury and increase the apoptosis of tubule epithelial cells under stress (19). These findings indicate that the regulation of mitochondrial dynamics may be one approach in protecting mitochondrial function and reducing tubule epithelial cell apoptosis. However, there have been no studies focusing on the mitochondria dynamics of tubule epithelial cells in septic AKI.

In the present ex vivo study, it was confirmed that excessive mitochondrial fission and insufficient mitochondrial fusion were present in LPS-induced HK-2 cells. Following stimulation with LPS, the HK-2 cells showed significantly increased expression of DAPK2, and decreases in the expression levels of Mfn1 and Mfn2, indicating an increase of mitochondrial fission and decrease of mitochondrial fusion. Decreased mitochondrial dysfunction and cell apoptosis were also detected, in addition to increased levels of cytochrome $c$, caspase- 3 andcaspase- 9 , and decreased levels of Bcl-2, Bcl-xL and PARP.

It has been shown previously that UTI can suppress the excessive generation of superoxide anion radicals, systemic inflammation, oxidative stress and endothelial injury caused by endotoxins (7), particularly against LPS-induced kidney injury (20). In a previous rat model of ischemia, UTI was shown to have protective effects on mitochondria in the kidney $(8,9)$.

In the present study, when co-treated with UTI, the HK-2 cells showed higher expression levels of Mfn1 and Mfn2, and lower expression levels of Drp1, suggesting a positive role of UTI in preventing excessive mitochondrial fission. Mitochondrial dysfunction and cell apoptosis were also decreased as a subsequent effect. As previous studies have already shown that oxidative stress and inflammation can induce disordered mitochondrial fission $(21,22)$, the present study hypothesized that the regulatory effect of UTI on mitochondrial dynamics may function by suppressing the oxidative and inflammatory damage caused by LPS. The decreased levels of IL-6, TNF- $\alpha$ and MDA, and the increased expression of SOD support this hypothesis.

Taken together, the results of the present study suggested that UTI protected human tubular epithelial cells from apoptosis by preventing the excessive mitochondrial fission induced by LPS. The effect may result from its antioxidant and anti-inflammatory effects. Future investigations are required to evaluate the effect of UTI on mitochondrial dynamics in an animal model of sepsis.

In conclusion, the present study showed that UTI prevented mitochondrial dysfunction caused by LPS by promoting mitochondrial fusion and limiting mitochondrial fission, thus reducing the apoptosis of LPS-induced HK-2 cells. This protective effect may be accomplished through decreasing the release of inflammatory cytokines and limiting oxidative activities.

\section{Acknowledgements}

This study was part of the Program of The National Natural Science Foundation of China (grant no. 81201452). 


\section{References}

1. Schrier RW and Wang W: Acute renal failure and sepsis. N Engl J Med 351: 159-169, 2004.

2. Russell JA: Management of sepsis. N Engl J Med 355: 1699-1713, 2006.

3. Gonzalez AS, Elguero ME, Finocchietto P, Holod S, Romorini L, Miriuka SG, Peralta JG, Poderoso JJ and Carreras MC: Abnormal mitochondrial fusion-fission balance contributes to the progression of experimental sepsis. Free Radic Res 48: 769-783, 2014.

4. Jendrach M, Mai S, Pohl S, Vöth M and Bereiter-Hahn J: Shortand long-term alterations of mitochondrial morphology, dynamics and mtDNA after transient oxidative stress. Mitochondrion 8: 293-304, 2008.

5. Binns OA, DeLima NF, Buchanan SA, Mauney MC, Cope JT, Thies SD, Shockey KS, Tribble CG and Kron IL: Neutrophil endopeptidase inhibitor improves pulmonary function during reperfusion after eighteen-hour preservation. J Thorac Cardiovasc Surg 112: 607-613, 1996.

6. Hirose J, Ozawa T, Miura T, Isaji M, Nagao Y, Yamashiro K, Nii A, Kato K and Uemura A: Human neutrophil elastase degrades inter-alpha-trypsin inhibitor to liberate urinary trypsin inhibitor related proteins. Biol Pharm Bull 21: 651-656, 1998.

7. Tanaka R, Fujita M, Tsuruta R, Fujimoto K, Aki HS, Kumagai K, Aoki T, Kobayashi A, Izumi T, Kasaoka S, et al: Urinary trypsin inhibitor suppresses excessive generation of superoxide anion radical, systemic inflammation, oxidative stress and endothelial injury in endotoxemic rats. Inflamm Res 59: 597-606, 2010.

8. Ueki M, Yokono S and Ogli K: Effects of ulinastation on rat renal energy metabolism and blood flow in hemorrhagic shock. J Anesth 9: 65-69, 1995.

9. Taie S, Yokono S, Ueki M and Ogli K: Effects of ulinastatin (urinary trypsin inhibitor) on ATP, intracellular $\mathrm{pH}$, andintracellular sodium transients during ischemia and reperfusion in the rat kidney in vivo. J Anesth 15: 33-38, 2001.

10. Ryan MJ, Johnson G, Kirk J, Fuerstenberg SM, Zager RA and Torok-Storb B: HK-2: An immortalized proximal tubule epithelial cell line from normal adult human kidney. Kidney Int 45: 48-57, 1994.

11. Lerolle N, Nochy D, Guérot E, Bruneval P, Fagon JY, Diehl JL and Hill G: Histopathology of septic shock induced acute kidney injury: Apoptosis and leukocytic infiltration. Intensive Care Med 36: 471-478, 2010.
12. Jacobs R, Honore PM, Joannes-Boyau O, Boer W, De Regt J, De Waele E, Collin V and Spapen HD: Septic acute kidney injury: The culprit is inflammatory apoptosis rather than ischemic necrosis. Blood Purif 32: 262-265, 2011.

13. Koçkara A and Kayataş M: Renal cell apoptosis and new treatment options in sepsis-induced acute kidney injury. Ren Fail 35: 291-294, 2013.

14. Nunnari J, Wong ED, Meeusen S and Wagner JA: Studying the behavior of mitochondria. Methods Enzymol 351: 381-393, 2002.

15. Palmer CS, Osellame LD, Stojanovski D and Ryan MT: The regulation of mitochondrial morphology: Intricate mechanisms and dynamic machinery. Cell Signal 23: 1534-1545, 2011.

16. Li P, Nijhawan D, Budihardjo I, Srinivasula SM, Ahmad M, Alnemri ES and Wang X: Cytochrome $\mathrm{c}$ and dATP-dependent formation of Apaf-1/caspase-9 complex initiates an apoptotic protease cascade. Cell 91: 479-489, 1997.

17. Tang WX, Wu WH, Qiu HY, Bo H and Huang SM: Amelioration of rhabdomyolysis-induced renal mitochondrial injury and apoptosis through suppression of Drp-1 translocation. J Nephrol 26: 1073-1082, 2013

18. Zhang L, Jiang F, Chen Y, Luo J, Liu S, Zhang B, Ye Z, Wang W, Liang $X$ and Shi W: Necrostatin-1 attenuates ischemia injury induced cell death in rat tubular cell line NRK-52E through decreased Drp1 expression. Int J Mol Sci 14: 24742-24754, 2013.

19. Gall JM, Wang Z, Liesa M, Molina A, Havasi A, Schwartz JH, Shirihai O, Borkan SC and Bonegio RG: Role of mitofusin 2 in the renal stress response. PLoS One 7: e31074, 2012.

20. Ueki M, Taie S, Chujo K, Asaga T, Iwanaga Y, Ono J and Maekawa N: Urinary trypsin inhibitor reduces inflammatory response in kidney induced by lipopolysaccharide. J Biosci Bioeng 104: 315-320, 2007.

21. Wu S, Zhou F, Zhang Z and Xing D: Mitochondrial oxidative stress causes mitochondrial fragmentation via differential modulation of mitochondrial fission-fusion proteins. FEBS J 278: 941-954, 2011.

22. Motori E, Puyal J, Toni N, Ghanem A, Angeloni C, Malaguti M, Cantelli-Forti G, Berninger B, Conzelmann KK, Götz M, et al: Inflammation-induced alteration of astrocyte mitochondrial dynamics requires autophagy for mitochondrial network maintenance. Cell Metab 18: 844-859, 2013. 